\title{
Edítorial.
}

\section{CORTICAL FUNCTION IN RELATION TO THE NEUROSES.}

W HATEVER the explanation of the genesis of the neuroses, there can be no question that many of the symptoms are the direct result of fatigue. Such fatigue may be produced by physical overwork, though this is rare, or by mental overwork on the conscious or at the unconscious level. Fatigue, however, is not an explanation of the neurasthenic state in itself. This is why the old-fashioned ' rest cure' in cases of neurasthenia was frequently inadequate. So long as the fatigue was due to friction between the ego as a whole and the outside world, then the removal of the friction by isolation of the ego allowed for the necessary recuperation, and recovery resulted. When, on the other hand, the fatigue was the result of friction within the ego, then mere rest was not enough, for the friction still persisted and cure did not ensue. It is a truism in neurology that the higher structures are more readily fatigued than those of lower levels ; naturally, the highest level structures suffer first and most severely from any process of fatigue.? The study of racial and individual development shows clearly that the highest grade structure in man is the cerebral cortex, and the functions of this aggregation of cells are upset by the processes of fatigue earlier and more markedly than the other functions of the body.

The conceptions of these functions have changed not a little in recent years. With the tremendous impetus given to anatomical and histological study by the improvements in optical and electrical methods which took place in the last half of the nineteenth century, it seemed possible that the researches of such workers as Ferrier and Wernicke would lead to a mapping out of the whole cortex into geographical areas, each of which would be found to correspond to a definite function connected with definite parts of the body. More recent work, however, has turned attention in other directions. Sherrington has shown that stimulation of certain spots even in such well-defined regions as the cortical motor area does not always produce the same result. He found that responses in the muscular system depend on such factors as the order of stimulation in the motor field ; for example, excitation from above downwards in a certain area results in elbow flexion, while from 
below upwards stimulation of the same points results in adduction of the thumb and extension of the index. Exact anatomical correspondence can no longer be regarded as part of the business of the cortex, though it is, of course, quite clear that certain cortical areas have to do with certain major functions of the body, such as motion, sensation, and the work of the distance-receptors involving vision and hearing, and so on. In these relations the functions to which cortical structure is essential are control, integration, discrimination and reference in time and space. In the motor area the first three are specially well illustrated. The work of Hughlings Jackson and his successors has shown how the uncontrolled action of lower motor centres is the real explanation of fits, while it is only when the motor cortex is intact that integrated and co-ordinated movements as opposed to muscular actions are possible. Again, without the intervention of this cortical function the discriminated movements of skilful manipulation are impossible. The work of Head and his coadjutors on sensation leads to similar conclusions. The sensory cortex subserves five functions: (1) the recognition of posture and passive movement; (2) the recognition of certain tactile elements ; (3) the appreciation of two points applied simultaneously to two different spots on the surface of the skin, and also the recognition of the size and shape of objects in contact with the skin (spatial discrimination); (4) the localization of the situation of a stimulated spot on the skin, and the recognition of two consecutive stimuli (discrimination of time); (5) the recognition and discrimination of a scale of sensations with heat at one end and cold at the other. In addition it is the function of the cortex to relate one sensation with another.

Bianchi's work on the frontal lobes suggests similar main principles of function. He finds that monkeys whose frontal lobes are mutilated show defects of perception, and of discrimination between objects with superficial resemblance; they cannot distinguish between threat and reality, and so become to a large extent incapable of play. They also display deficient observation, objects which would be investigated by the normal monkey being overlooked. Memory is defective, so that past experiences are not utilized, nor do they learn by present experience. Associative power is greatly reduced, and there are no new adaptations. Judgment is poor and immediate. There is a lack of initiative, and one movement does not lead to another; thus a monkey will seize a door handle (stimulus to bright object), but will not proceed to turn it. The primitive emotions remain - the desire for satisfaction of hunger, thirst and other organic needs, and especially that irrational, illogical fear which seems to have no definite relation to stimuli ; but higher sentiments, such as friendship, gratitude, jealousy maternal protection, dominion, authority, self-esteem, ridicule, and, above all, that of 
sociality, disappear. Conduct is incoherent, while stereotyped actions and tics are often present.

It is remarkable that the behaviour of neurotics is mainly characterized by deficiency in what we have learnt to regard as the chief functions of the cortex. To take a few examples : in hysterical paralyses it is movements that are impeded, not individual muscular actions. It is characteristic of hysterical pain that the reaction in some ways resembles that seen in the thalamic syndrome, in which cortical control is absent. The behaviour of neurotics always tends to be of the 'all-or-none' variety. This is specially true of their emotional reactions, which closely resemble those of Bianchi's monkeys. Their undiscriminated anxieties and fears are primitive affective reactions in no way co-ordinated into properly organized emotions and sentiments, and the resolution of these by the process of abreaction essentially involves their proper discrimination and correlation. In the case of the obsessions the salient feature is the want of accurate reference in time and space. So much is this so that it is only by restoring the proper timespace-relationship of these symptoms that they can be removed.

This brief review may serve to indicate that the immediate precursor of neurotic symptoms is the abrogation of cortical function, and that this failure of function is probably due to the action of fatigue products. This conception does not run counter to other explanations of neurotic symptoms, such as dissociation, for the most marked forms of dissociation of function are those produced when the associative function of the cortex is in abeyance. Indeed, it is clear that a properly associated personality is only possible when the cortex is intact. It is, of course, the duty of the physician not to be content with this explanation of symptoms, but to strive to discover the true cause of the fatigue ; by this means only will he cure the disease, and not merely remove the symptoms. None the less, the recognition that fatigue of the cortex is an important factor in this connection is salutary, since it will tend to broaden the outlook of the therapist and prevent his applying only one method of treatment to all cases of neuroses. He will remember that fatigue may be induced by mental conflict, by prolonged toxæmia, by endocrine disturbances, and by overwork, and so he is less likely to miss his view of the wood by too great concentration on individual trees. 